\title{
Impossibility Results for Universal Composability in Public-Key Models and with Fixed Inputs*
}

\author{
Dafna Kidron ${ }^{\dagger} \quad$ Yehuda Lindell ${ }^{\ddagger}$
}

June 6, 2010

\begin{abstract}
Universal composability and concurrent general composition consider a setting where secure protocols are run concurrently with each other and with arbitrary other possibly insecure protocols. Protocols that meet the definition of universal composability are guaranteed to remain secure even when run in this strongly adversarial setting. In the case of an honest majority, or where there is a trusted setup phase of some kind (like a common reference string or the keyregistration public-key infrastructure of Barak et al. in FOCS 2004), it has been shown that any functionality can be securely computed in a universally composable way. On the negative side, it has also been shown that in the plain model where there is no trusted setup at all, there are large classes of functionalities which cannot be securely computed in a universally composable way without an honest majority.

In this paper we extend these impossibility results for universal composability. We study a number of public-key models and show for which models the impossibility results of universal composability hold and for which they do not. We also consider a setting where the inputs to the protocols running in the network are fixed before any execution begins. The majority of our results are negative and we show that the known impossibility results for universal composability in the case of no honest majority extend to many other settings.
\end{abstract}

Keywords: universal composability, impossibility results, concurrent general composition, publickey models.

\footnotetext{
*This research was partially supported by the ISRAEL SCIENCE FOUNDATION (grant No. 781/07).

${ }^{\dagger}$ Department of Computer Science, Bar-Ilan University, Israel. Email: dafna.kidron@gmail.com

${ }^{\ddagger}$ Department of Computer Science, Bar-Ilan University, Israel. Email: Iindell@cs.biu.ac.il
} 


\section{Contents}

1 Introduction 1

2 Preliminaries $\quad 5$

2.1 Brief Overview of Universal Composability . . . . . . . . . . . . . . 5

2.2 The Impossibility Results of $[12] \ldots \ldots \ldots \ldots \ldots$

3 UC-Security in Public-Key Models $\quad 8$

3.1 Bulletin-Board Certificate Authority $(\mathrm{CA}) \ldots \ldots \ldots \ldots$

3.2 Bulletin-Board CA with Independent Keys . . . . . . . . . . . . . . . . . . . 14

3.3 The Bare Public-Key Model . . . . . . . . . . . . . . . . . . . . 16

3.4 A Strong Bare Public-Key Model . . . . . . . . . . . . . . . . . . . . . 17

3.5 Active Key Registration by the CA . . . . . . . . . . . . . . . 18

3.6 CA Corruptions and UC Security . . . . . . . . . . . . . . . . . . 19

3.6.1 Passive (Semi-Honest) CA Corruptions . . . . . . . . . . . . . . 19

3.6 .2 Indistinguishable Malicious CA Corruptions . . . . . . . . . . . . . . 20

4 Universal Composability with Fixed Inputs 20 


\section{Introduction}

In the setting of secure multiparty computation, a set of parties with private inputs wish to jointly compute some functionality of their inputs. Loosely speaking, the security requirements of such a computation are that nothing is learned from the protocol other than the output (privacy), and that the output is distributed according to the prescribed functionality (correctness). More exactly, the result of an execution of a secure protocol must be like the result of an "ideal execution" with an incorruptible trusted party who honestly computes the function for the parties (cf. [7] or [19, Section 7.1]). These security requirements must hold in the face of a malicious adversary who controls some subset of the parties and can arbitrarily deviate from the protocol instructions. Powerful feasibility results have been shown for this problem in both the information-theoretic and computational settings $[32,20,6,13]$. In the computational setting, it has been shown that any multiparty probabilistic polynomial-time functionality can be securely computed for any number of corrupted parties, assuming the existence of enhanced trapdoor permutations [32, 20, 19].

Security under concurrent composition. The above-described feasibility results relate only to the stand-alone setting, where a single protocol is run in isolation. However, in modern network settings, protocols must remain secure even when many protocol executions take place concurrently and are being attacked in a coordinated manner. Informally speaking, a protocol is said to be secure under concurrent general composition if it is secure when run many times concurrently, alongside other secure and insecure protocols. The question of what can and cannot be securely computed in this strongly adversarial setting has been the topic of much research over recent years. In this paper, we focus on the framework of universal composability [8]; this framework presents a definition of security with the important property that any protocol meeting the definition is guaranteed to remain secure under concurrent general composition. Such protocols are called UC-secure for short.

It has been shown that UC-secure protocols exist for essentially any functionality in the case of an honest majority [8], or where there is a common reference string [14] or an active key-registration functionality [2]. Thus, in these cases, the same broad feasibility results of the stand-alone model hold (except that in the stand-alone model, neither an honest majority nor a trusted setup phase is needed). When considering the case of no honest majority and no trusted setup in the setting of universal composability, the situation is completely different. Specifically, it has been shown that in such a setting, large classes of functionalities cannot be UC realized $[10,8,12,17]$. Due to this, a search has been initiated to find alternative models and definitions of security for this setting; see for example $[30,31,22,4]$.

Our results - public-key models. In this paper, we extend the broad impossibility results of [12]. The UC impossibility results proven in [12] hold for the plain model (where there is no trusted setup whatsoever) and for the case of no honest majority. However, they do not consider the case that some basic public-key infrastructure may be in place. This is especially serious because standard secure computation can only really be carried out when there are authenticated channels (see [1] for a study of this issue), and in practice such authenticated channels are implemented using a public-key infrastructure. Thus, the actual impossibility results of [12] do not cover the most interesting setting where a basic public-key infrastructure is used for obtaining authenticated channels. The public-key infrastructure needed for obtaining authenticated channels was shown in [9] to be a basic bulletin-board functionality (i.e., this functionality has the property that any party can register any key and no checks are carried out on the registered key; the only guarantee is that a key that is retrieved for a certain party is indeed the same as what was registered). We call 
the ideal functionality that implements this bulletin board the bulletin-board $C A$, denoted $\mathcal{F}_{\mathrm{BBCA}}$. We use $\mathcal{F}_{\mathrm{BBCA}}$ as our starting point and show that all of the impossibility results of [12] actually hold in the $\mathcal{F}_{\mathrm{BBCA}}$-hybrid model as well (i.e., impossibility carries over even when all parties have access to the ideal functionality that implements $\left.\mathcal{F}_{\mathrm{BBCA}}\right)$. That is, we prove the following:

Impossibility with only a bulletin-board CA: There exist large classes of deterministic twoparty functionalities that cannot be $U C$ realized by any protocol, even in the $\mathcal{F}_{\mathrm{BBCA}}$-hybrid model.

Our proof works by showing that the main lemma of [12] can be adapted to hold even when the parties are given access to the ideal functionality $\mathcal{F}_{\mathrm{BBCA}}$. This lemma shows that there exists a successful split adversarial strategy for every UC-secure two-party protocol. Loosely speaking, this strategy means that it is possible for an adversary to extract the honest party's input and also bias the output that the honest party receives. This clearly implies that many (if not most) functionalities cannot be UC realized. The importance of this result is in showing that UC security is also impossible to achieve in the realistic model where a public-key infrastructure is in place for obtaining authenticated channels. We remark that impossibility holds even if $\mathcal{F}_{\mathrm{BBCA}}$ enforces all keys to be unique.

Before proceeding to describe our other results, one remark is in order. In reality, the $\mathcal{F}_{\mathrm{BBCA}}$ ideal functionality would typically be implemented using digital certificates and a public key of one or more certificate authorities. Could we not just use the public key of the CA as a common reference string and thereby construct UC-secure protocols for any functionality using the result of [14]? We argue that this is not the case. This is mainly due to the fact that the level of trust needed from an authority choosing a common reference string is far higher than that needed from a CA. In order to see this observe that the party choosing the common reference string may be able to learn all of the parties' inputs over all executions by simply passively eavesdropping on the communication. Indeed, a close look at protocols in this model demonstrates that this is usually the case. In contrast, a CA who posts false keys for honest parties must carry out an active attack in every protocol execution. Furthermore, even if it does so, it can learn whatever a man-in-themiddle attacker can learn in an unauthenticated channels setting, which is rather limited; see [1] for more discussion on this. We conclude that although implementing $\mathcal{F}_{\mathrm{BBCA}}$ requires some trust, and this trust in reality also boils down to some "string", there is a fundamental difference between trusting a public key that can be used for achieving authenticated channels and trusting a common reference string. Thus, it would be highly desirable to have UC-secure protocols in the $\mathcal{F}_{\mathrm{BBCA}}$-hybrid model. Unfortunately, this is ruled out by the aforementioned result.

Having considered this basic CA functionality, we study stronger versions with the aim of drawing the line between feasibility and impossibility, and of clarifying what is needed to bypass the impossibility. In addition to the impossibility result described above, we obtain the following informally stated results:

1. Feasibility for bulletin-board CA with independent keys: We consider a further strengthening of the CA to one that prevents any party from retrieving a public key from the bulletin board before all parties have registered their keys. This assumes that the registered keys are kept secret during registration. Note that this forces the parties' keys to be independent of each other because no party can see any other party's registered key before it registers its own. We show that UC secure protocols can be constructed in this model. (In fact this is very easy to achieve by simply observing that it is possible to securely toss coins in this model.)

2. Impossibility for the bare public-key model: A popular public-key model that has been used in a number of settings in cryptography is the "bare" public-key model. In this model, the 
CA is the same as the bulletin-board CA except that all keys must be registered before any execution of the secure protocol begins. We note that there is no limitation on the arbitrary other protocols that may run during the registration phase. ${ }^{1}$ In this case, we show that once again, the UC impossibility results carry over. This is of special interest because this model has recently be used to achieve stronger notions of non-malleable concurrent zero-knowledge $[18,29]$. It seems that the aim of this direction is to eventually achieve UC security (or equivalently, security under concurrent general composition) in this model. We show that the security achieved in this model must fall short of UC security.

3. Feasibility for a strong bare public-key model: We observe that if the bare public-key model is strengthened so that no protocols whatsoever are run during the registration phase, then it is possible to run a coin-tossing protocol that is secure in the stand-alone model in order to construct a common reference string (and thereby achieve UC security [14]). The reason that it suffices to consider the stand-alone model for the coin-tossing is due to the fact that no protocols whatsoever are run during this period. Thus this strengthening trivially enables UC security; unfortunately, we view it as highly unrealistic.

4. Feasibility for active key registration by the $C A$ : We analyze the key-registration functionality used by [2] to achieve UC security and show where the proof of impossibility fails with respect to the functionality. This highlights what properties of the functionality are used to bypass the impossibility results.

In addition to the above, we study what happens when the CA may be partially corrupted (if it is fully corrupted, then the UC impossibility results clearly hold, irrespective of what the functionality does). We show the following:

1. Passive $C A$ corruptions: we observe that if the CA functionality behaves in a semi-honest way and reveals its internal state to the adversary (but otherwise acts honestly), then UC security can be achieved. This follows immediately from the fact that the CA can generate a uniformly distributed common reference string (in which case there is no hidden internal state), and thus the protocol of [14] can be used.

2. Indistinguishable malicious CA corruptions: we study what happens if the CA behaves maliciously, yet generates messages that are indistinguishable from those generated by an honest CA. We show that in this case the UC impossibility results also hold, irrespective of how the $\mathrm{CA}$ is defined.

We believe that our results provide a comprehensive study of UC feasibility in public-key models. It is our hope that they make sense out of the confusing myriad of public-key models that are in the literature. (Of course, we do not claim to have covered all possible public-key models, nor all that appear in the literature. Nevertheless, we hope that given the results here, an analysis of other models is relatively simple.) Our results are summarized in the following table:

\footnotetext{
${ }^{1}$ Typically, the bare public-key model was considered for self composition where the only protocol running is the secure one. Thus the issue of arbitrary other protocols was not raised. We interpret the bare public-key model in the context of concurrent general composition in this way because we view it as a far more realistic model. The interpretation whereby no protocol whatsoever is being run during the registration phase is considered next (and we call it the "strong" bare public-key model).
} 


\begin{tabular}{|c|c|c|}
\hline The Model & The Result & Notes \\
\hline Bulletin-board CA & Impossibility holds & Used by $[9]$ \\
Bulletin-board with independent keys & UC security achievable & Generate a CRS and use [14] \\
Bare public-key model & Impossibility holds & A popular model \\
Strong bare public-key model & UC security achievable & Not a realistic model \\
Active key registration & UC security achievable & Result shown in $[2]$ \\
Passive CA corruptions & UC security achievable & Generate a CRS and use [14] \\
Indistinguishable malicious CA corruptions & Impossibility holds & - \\
\hline
\end{tabular}

Concurrent general composition with fixed inputs. In addition to the above study on UC security in public-key models we ask another question that relates to the possibility of obtaining security under concurrent general composition. This question is concerned with how the parties' inputs are chosen. It has been shown that when the honest parties chooses their inputs adaptively, ${ }^{2}$ then for a large class of functionalities (in fact, most functionalities), security under concurrent self composition $^{3}$ is equivalent to security under concurrent general composition [27]. Therefore all of the impossibility results that hold for concurrent general composition also hold for concurrent self composition with adaptively chosen inputs. This equivalence does not hold when the inputs are all fixed ahead of time (i.e., where the honest parties receive a vector specifying the input for each execution). The fact that there exist protocols that are secure under concurrent self composition with fixed inputs but not with adaptively chosen inputs was demonstrated in [23]. Later, this separation was shown to hold even for the zero-knowledge functionality. That is, it has been shown that it is possible to construct zero-knowledge protocols that are secure under concurrent self composition with arbitrary roles (meaning that players can simultaneously be provers and verifiers), as long as the inputs are all fixed before any execution begins [3]. We stress that such a construction is impossible to achieve when inputs are adaptively chosen. Thus, it is strictly easier to achieve concurrent self composition with fixed inputs than it is to achieve with adaptively chosen inputs. We ask the following question:

For what functionalities is it possible to construct protocols that remain secure when run once concurrently together with an arbitrary other protocol, and the inputs are fixed before any execution begins.

We call this setting minimal concurrent general composition with fixed inputs (it is minimal in the sense that there are only two protocol executions). We remark that it has already been shown that when inputs may be adaptively chosen, broad impossibility holds even if there are only two executions as above [26]. The novelty in the question here is therefore the fact that the inputs are a priori fixed. We show the following:

Impossibility for fixed inputs: There exist large classes of deterministic two-party functionalities that cannot be securely realized by any protocol under minimal concurrent general composition with fixed inputs.

We prove this theorem by defining a variant of the UC model where the environment first writes the inputs to all parties, and only then does the execution begin. We then show that security under minimal concurrent general composition with fixed inputs implies this UC variant, and finally that all of the impossibility results of [12] hold for this variant.

\footnotetext{
${ }^{2}$ This adaptive choice of inputs means that the inputs used by honest parties may be determined as a function of the outputs that they have already received in previous executions that have concluded. We stress that it is always assumed that the adversary can chooses its inputs adaptively. The question of interest here is with respect to the honest parties.

${ }^{3}$ In the setting of concurrent self composition, a single protocol is run many times concurrently.
} 
Subsequent work. The question of finding a "minimal" setup assumption for UC security has been studied recently in [16]. They show that it suffices to have a public-key infrastructure where each party has "some knowledge" of a secret associated with their public key. Their model differs from our bulletin-board and bare public-key models in this addition of guaranteed knowledge of a secret. Thus, they manage to avoid the possibility results with this assumption. Another work of relevance is that of [24] who provide a general framework for modeling different setup assumptions for achieving UC security.

\section{Preliminaries}

\subsection{Brief Overview of Universal Composability}

We present a very brief overview of how security is defined in the UC framework. See [8] for further details. As in other general definitions (e.g., [21, 28, 5, 7]), the security requirements of a given task (i.e., the functionality expected from a protocol that carries out the task) are captured via a set of instructions for a "trusted party" that obtains the inputs of the participants and provides them with the desired outputs. Informally, a protocol securely carries out a given task if running the protocol with a real adversary amounts to "emulating" an ideal process in which the parties hand their inputs to a trusted party who computes the appropriate functionality and hands their outputs back, without any other interaction. We call the algorithm run by the trusted party the ideal functionality, and describe the interaction in the ideal model to be between the parties and the ideal functionality (with the understanding that what we really mean is the trusted party running this functionality).

In order to prove the universal composition theorem, the notion of emulation in this framework is considerably stronger than in previous ones. Traditionally, the model of computation includes the parties running the protocol and an adversary $\mathcal{A}$ that controls the communication channels and potentially corrupts parties. "Emulating an ideal process" means that for every adversary $\mathcal{A}$ there should exist an "ideal process adversary", or simulator, $\mathcal{S}$ such that the distribution over all parties' inputs and outputs is essentially the same in the ideal and real processes. In the UC framework, an additional entity, called the environment $\mathcal{Z}$, is introduced. The environment generates the inputs to all parties, reads all outputs, and in addition interacts with the adversary in an arbitrary way throughout the computation. A protocol is said to UC realize a given ideal functionality $\mathcal{F}$ if for any "real-life" adversary $\mathcal{A}$ that interacts with the protocol and the environment there exists an

"ideal-process adversary" $\mathcal{S}$, such that no environment $\mathcal{Z}$ can tell whether it is interacting with $\mathcal{A}$ and parties running the protocol, or with $\mathcal{S}$ and parties that interact with $\mathcal{F}$ in the ideal process. In a sense, here $\mathcal{Z}$ serves as an "interactive distinguisher" between a run of the protocol and the ideal process with access to $\mathcal{F}$. A bit more precisely, Let $\operatorname{REAL}_{\pi, \mathcal{A}, \mathcal{Z}}$ be the ensemble describing the output of environment $\mathcal{Z}$ after interacting with parties running protocol $\pi$ and with adversary $\mathcal{A}$. Similarly, let IDEAL $\mathcal{F}, \mathcal{S}, \mathcal{Z}$ be the ensemble describing the output of environment $\mathcal{Z}$ after interacting in the ideal process with adversary $\mathcal{S}$ and parties that have access to the ideal functionality $\mathcal{F}$. We note that all entities run in time that is polynomial in the security parameter, denoted by $k$. In addition, the environment receives an initial input $z$, and security is required to hold for all such inputs (this makes the environment a non-uniform machine). Security in the UC framework is formalized in the following definition.

Definition 2.1 Let $\mathcal{F}$ be an ideal functionality and let $\pi$ be a two-party protocol. We say that $\pi$ $\mathrm{UC}$ realizes $\mathcal{F}$ if for every adversary $\mathcal{A}$ there exists an ideal-process adversary $\mathcal{S}$ such that for every environment $\mathcal{Z}$, the ensembles $\operatorname{IDEAL} \mathcal{F}, \mathcal{S}, \mathcal{Z}$ and $\operatorname{REAL}_{\pi, \mathcal{A}, \mathcal{Z}}$ are indistinguishable. 
Variants of the UC definition. As with the results of [12], our results hold for all known variants of the UC definition and are resilient to changes in definition of polynomial-time, the order of activations and so on. Nevertheless, in order to write our proofs we need to specify a model. We take the model where all messages are sent via the adversary, including the messages that are sent between parties and the ideal functionalities. In order to model private values that may be sent between the parties and functionalities, we specify that these messages are composed of a public header and possibly private contents (although in this work there will only be one functionality that has private contents; all others are completely public). This convention was used in [14] (see [25, Page 97]) and can be modeled in the regular UC framework (where messages are sent directly and privately between honest parties and the ideal functionalities), by defining a canonical form for the ideal functionality that always asks the adversary when to receive a message and when to send it (and the query is based on sending the public header). We remark that the adversary cannot modify messages sent between parties and the ideal functionality. The question of whether it can or cannot modify messages sent between honest parties is of no relevance here because we always consider the scenario where there are two parties, one of which is corrupted. For a full detailed description of the exact UC definition that we use here, see [25].

Non-trivial protocols and the requirement to generate output. As we have mentioned above, in the variant of UC that we consider here, the ideal-process adversary can choose when (if ever) to deliver messages that are sent between the parties and the ideal functionality. Consequently, the definition provides no guarantee that a protocol will ever generate output or "return" to the calling protocol. Rather, the definition concentrates on the security requirements in the case that the protocol generates output.

A corollary of the above fact is that a protocol that "hangs", never sends any messages and never generates output, UC realizes any ideal functionality. However, such a protocol is clearly not interesting. We therefore use the notion of a non-trivial protocol [14]. Such a protocol has the property that if the real-life adversary delivers all messages and does not corrupt any parties, then the ideal-process adversary also delivers all messages (and does not corrupt any parties). Thus, non-trivial protocols have the minimal property that when all participants are honest (and the adversary does not prevent any messages from being delivered), then all parties receive output. Again, as with [12], our impossibility results are for non-trivial protocols only.

The UC composition theorem. As mentioned, a universally composable protocol remains secure under a very general composition operation. In particular, it maintains its security even when run concurrently with other arbitrary protocols that are being run by arbitrary sets of possibly different sets of parties, with possibly related inputs. Thus, universally composable protocols can be used in modern networks, and security is guaranteed. It is therefore of great importance to understand what functions can and cannot be UC realized under this definition. See [8] for more details.

\subsection{The Impossibility Results of [12]}

The impossibility results of [12] are obtained by proving a lemma that describes an "attack" that is possible against any two-party UC-secure protocol that securely realizes a deterministic function $f$. The lemma is then used to derive a series of impossibility results for different classes of functions. The lemma refers to deterministic, polynomial-time computable functions $f: X \times X \rightarrow\{0,1\}^{*} \times$ $\{0,1\}^{*}$, where $X \subseteq\{0,1\}^{*}$ is an arbitrary, possibly infinite, domain (for simplicity it is assumed 
that both parties' inputs are from the same domain, but this makes no difference). The functions considered have two outputs, one for each party and are denoted $f=\left(f_{1}, f_{2}\right)$ where $f_{1}$ denotes the first party's output and $f_{2}$ denotes the second party's output.

Motivation. The idea behind the main lemma of [12] is as follows. An ideal-model simulator in the UC model works by interacting with an ideal functionality; namely, it sends the functionality an input (in the name of the corrupted party) and receives back an output. Since the simulated view of the corrupted party is required to be indistinguishable from its view in a real execution, it must hold that the input sent by the simulator to the ideal functionality corresponds to the input that the corrupted party (implicitly) uses. Furthermore, the corrupted party's output from the protocol simulation must correspond to the output received by the simulator from the ideal functionality. That is, such a simulator must be able to "extract" the input used by the corrupted party, in addition to causing the corrupted party to output a value that corresponds to the output received by the simulator from the ideal functionality.

The main point behind the lemma of [12] is the observation that in the plain model, a malicious adversary in the real model can do "whatever" the simulator can do. Thus, since the simulator can extract the adversary's input, a real adversary can extract the honest party's input in a real execution (something that should not be possible in a secure protocol). In other models of secure computation and when some trusted setup assumptions are used, this attack cannot be carried out because the simulator typically has some additional "power" that a malicious party does not. (In stand-alone secure computation, this power is usually the ability to rewind the adversary, something that cannot be done to a real party. In the UC model with setup assumptions like a common reference string, this power is the ability to choose the string and make it not necessarily uniform.)

Split adversarial strategies. We describe the notion of a split adversarial strategy for a corrupted $P_{2}$ as used in [12]. In our results on impossibility results for public-key models, we will prove exactly the same lemma except that we will show that it holds in some public-key models (rather than in the plain model).

Intuitively, the adversarial strategy that is constructed for a malicious $P_{2}$ is one that consists of two separate machines: $P_{2}^{a}$ and $P_{2}^{b}$. Entity $P_{2}^{a}$ interacts with (the honest) $P_{1}$ and its aim is to "extract" the input used by the honest $P_{1}$ (it actually does this by running the ideal simulator for the protocol who, as we have mentioned, is able to extract such inputs). In contrast, entity $P_{2}^{b}$ emulates the ideal functionality for the simulator that is run by $P_{2}^{a}$. Loosely speaking, $P_{2}^{a}$ first "extracts" the input used by $P_{1}$. Entity $P_{2}^{a}$ then hands this input to $P_{2}^{b}$, who computes the function output and hands it back to $P_{2}^{a}$. Entity $P_{2}^{a}$ then continues with the emulation, and causes $P_{1}$ to output a value that is consistent with the input that is chosen by $P_{2}^{b}$ (this last step must also be carried it in any ideal simulation, and so once again can also be achieved by an attack in a real execution). The formal definition of this strategy appears below. We first present the "structure" of the attack and then what it means to be "successful".

Definition 2.2 (split adversarial strategy): Let $f: X \times X \rightarrow\{0,1\}^{*} \times\{0,1\}^{*}$ be a polynomial-time function where $f_{1}$ and $f_{2}$ denote the first and second outputs of $f$, respectively, and let $\pi_{f}$ be a protocol. Let $X_{2} \subseteq X$ be a polynomial-size subset of inputs (i.e., $\left|X_{2}\right|=\operatorname{poly}(k)$, where $k$ is the security parameter), and let $x_{2} \in X_{2}$. Then, a corrupted party $P_{2}$ is said to run a split adversarial strategy if it consists of machines $P_{2}^{a}$ and $P_{2}^{b}$ such that:

1. Upon input $\left(X_{2}, x_{2}\right)$, party $P_{2}$ internally gives the machine $P_{2}^{b}$ the input pair $\left(X_{2}, x_{2}\right)$. 
2. An execution between (an honest) $P_{1}$ running $\Pi_{f}$ and $P_{2}=\left(P_{2}^{a}, P_{2}^{b}\right)$ works as follows:

(a) $P_{2}^{a}$ interacts with $P_{1}$ according to some specified strategy.

(b) At some stage of the execution $P_{2}^{a}$ hands $P_{2}^{b}$ a value $x_{1}^{\prime}$.

(c) When $P_{2}^{b}$ receives $x_{1}^{\prime}$ from $P_{2}^{a}$, it computes $y_{1}^{\prime}=f_{1}\left(x_{1}^{\prime}, x_{2}^{\prime}\right)$ for some $x_{2}^{\prime} \in X_{2}$ of its choice (chosen according to any efficient strategy).

(d) $P_{2}^{b}$ hands $P_{2}^{a}$ the value $y_{1}^{\prime}$, and $P_{2}^{a}$ continues interacting with $P_{1}$.

Informally speaking, a split adversarial strategy is said to be successful if the value $x_{1}^{\prime}$ procured by $P_{2}^{a}$ is "equivalent to" (the honest) $P_{1}$ 's input $x_{1}$ with respect to $f_{2}$. That is, the output of $P_{2}$, when computed according to $f_{2}$ and when $P_{2}$ has input $x_{2}^{\prime} \in X_{2}$, is the same whether $x_{1}$ or $x_{1}^{\prime}$ is used. (Note that $x_{1}^{\prime}$ may differ from $x_{1}$ with respect to $P_{1}$ 's output, but only the effect on $P_{2}$ 's output is considered.) Furthermore, $P_{2}^{a}$ should succeed in causing $P_{1}$ to output the value $y_{1}=f_{1}\left(x_{1}, x_{2}^{\prime}\right)$. That is, the output of $P_{1}$ should be consistent with the value $x_{2}^{\prime}$ chosen by $P_{2}^{b}$.

Definition 2.3 (successful strategies): Let $f$ be a polynomial-time function and $\pi_{f}$ a protocol, as in Definition 2.2. Furthermore, let $k$ be the security parameter and let $\mathcal{Z}$ be an environment who hands an input $x_{1} \in X$ to $P_{1}$ and a pair $\left(X_{2}, x_{2}\right)$ to $P_{2}$, where $X_{2} \subseteq X,\left|X_{2}\right|=\operatorname{poly}(k)$, and $x_{2} \in_{R} X_{2}$. Then, a split adversarial strategy for a malicious $P_{2}$ is said to be successful if for every $\mathcal{Z}$ as above and every input $z$ to $\mathcal{Z}$, the following two conditions hold in a real execution of $P_{2}$ with $\mathcal{Z}$ and an honest $P_{1}$ :

1. The value $x_{1}^{\prime}$ output by $P_{2}^{a}$ in step $2 b$ of Definition 2.2 is such that for every $x_{2} \in X_{2}$, $f_{2}\left(x_{1}^{\prime}, x_{2}\right)=f_{2}\left(x_{1}, x_{2}\right)$.

2. $P_{1}$ outputs $f_{1}\left(x_{1}, x_{2}^{\prime}\right)$, where $x_{2}^{\prime}$ is the value chosen by $P_{2}^{b}$ in step $2 c$ of Definition 2.2 .

It is proven in [12] that a successful split adversarial strategy exists for any protocol that UC realizes a two-party function in the plain model.

\section{UC-Security in Public-Key Models}

In this section we investigate the question of whether or not it is possible to achieve UC-security when there is some type of public-key infrastructure (but there is no honest majority). In its basic form, a public-key infrastructure is a type of "bulletin board" where public keys are published (together with the identity of their owner) and can be retrieved by all parties in a trusted way. (By trust here, we mean that we assume that an adversary cannot modify published keys or tamper with a public-key while it is being retrieved by an honest party.) One of the central questions that arises when considering such an infrastructure is the role of the Certificate Authority (CA) who accepts keys and posts them on the bulletin board. For example, we may consider a very basic public-key model where the CA receives keys without any conditions and posts them, and we may consider a public-key model where the CA requires the users to send the secret-key that is associated with the public-key to be published. As we have discussed in the introduction, we study a number of different models, most of which have appeared in the literature in the past. For each model, we investigate the possibility of obtaining UC-secure protocols in a hybrid model in which the CA is modeled by an ideal functionality. Our proofs of impossibility are achieved by reproving the main lemma of [12] in the different models. 


\subsection{Bulletin-Board Certificate Authority (CA)}

We prove broad impossibility results for achieving UC security, even when the protocol may use an ideal functionality that implements a basic bulletin-board CA. The functionality that we define for this CA carries out no checks on the keys that are registered. The only limitation is that each party can register at most one key (this actually makes no difference and our proofs carry through even if parties can register different keys under different session identifiers or sub-session identifiers). See Figure 1 for a formal description of the functionality.

\section{Functionality $\mathcal{F}_{\mathrm{BBCA}}$}

$\mathcal{F}_{\text {BBCA }}$ proceeds as follows, running with parties $P_{1}, P_{2}, \ldots$ and an adversary $\mathcal{S}$ :

- Register commands: Upon receiving a message (register, sid, $v$ ) from some party $P_{i}$, the functionality checks that no pair $\left(P_{i}, v^{\prime}\right)$ is already recorded. If this is the case, it records the pair $\left(P_{i}, v\right)$. Otherwise, it ignores the new message.

- Retrieve commands: Upon receiving a message (retrieve, sid, $P_{i}$ ) from some party $P_{j}$ or the adversary $\mathcal{S}$, the functionality checks if some pair $\left(P_{i}, v\right)$ is recorded. If yes, and $v$ is the recorded value, it sends $\left(\operatorname{sid}, P_{i}, v\right)$ to $P_{j}($ or $\mathcal{S})$. Otherwise, it returns $\left(\operatorname{sid}, P_{i}, \perp\right)$.

Figure 1: The Bulletin-Board CA functionality

Note that $\mathcal{F}_{\mathrm{BBCA}}$ carries out no checks on the format of the key, and also does not prevent parties from copying keys from other parties. Thus, this is arguably the most basic and simple type of CA that one could imagine. Nevertheless, this does not mean that it is useless. In fact, this is far from true as it suffices for obtaining authenticated channels [9]. Recall that authenticated channels are almost always assumed for secure protocols and thus an assumption like the existence of $\mathcal{F}_{\mathrm{BBCA}}$ is actually needed anytime that secure protocols are to be used.

We prove that the split adversarial strategy lemma of [12] holds in the $\mathcal{F}_{\mathrm{BBCA}}$-hybrid model, thereby implying that all of the impossibility results of [12] for deterministic functionalities hold also in this model. See Section 2.2 for the definition of a successful split adversarial strategy and for the intuition behind the lemma.

Lemma 3.1 Let $f$ be a polynomial-time two-party function, and let $\mathcal{F}_{f}$ be the two-party ideal functionality that receives $x_{1}$ from $P_{1}$ and $x_{2}$ from $P_{2}$, and hands them back their respective outputs $f_{1}\left(x_{1}, x_{2}\right)$ and $f_{2}\left(x_{1}, x_{2}\right)$. If $\mathcal{F}_{f}$ can be $U C$-realized in the $\mathcal{F}_{\mathrm{BBCA}}$-hybrid model by a non-trivial protocol $\pi_{f},{ }^{4}$ then there exists a machine $P_{2}^{a}$ such that for every machine $P_{2}^{b}$ of the form described in Definition 2.2, the split adversarial strategy for $P_{2}=\left(P_{2}^{a}, P_{2}^{b}\right)$ is successful, except with negligible probability.

Proof: The proof is similar to the proof of the analogous lemma in [12] with the appropriate changes made due to the fact that now we are working in the $\mathcal{F}_{\mathrm{BBCA}}$-hybrid model rather than the plain model. As we have mentioned in Section 2.2 the basic idea is that if $\mathcal{F}_{f}$ can be UC realized by a protocol $\pi_{\mathcal{F}}$, then this implies the existence of an ideal-process adversary (or simulator) $\mathcal{S}$ that can extract the input used by $\mathcal{A}$. (It must be able to extract this input in order to send it to the ideal functionality.) The key point in the proof is that $\mathcal{S}$ must essentially accomplish this

\footnotetext{
${ }^{4}$ Recall that a non-trivial protocol is such that if the real model adversary corrupts no party and delivers all messages, then so does the ideal model adversary. This rules out the trivial protocol that does not generate output. See Section 2.1 for details.
} 
extraction while running a "straight-line black-box" simulation, meaning that it cannot rewind $\mathcal{A}$ and also has no access to its code. Stated differently, $\mathcal{S}$ interacts with $\mathcal{A}$ just like real parties interact in a protocol execution. We remark that technically, $\mathcal{S}$ is able to rewind $\mathcal{A}$ and inspect its code. However, we will construct a specific $\mathcal{A}$ and $\mathcal{Z}$ for which these capabilities are rendered useless. Now, if $\mathcal{S}$ can extract $\mathcal{A}$ 's input by interacting with it like in a real execution, and if $\mathcal{A}$ behaves like an honest party (which will be the case, as will be shown), then this means that $\mathcal{S}$ can extract an honest party's input in an honest interaction. The next step from this observation is that $\mathcal{S}$ can actually be used by an adversary to extract an honest party's input (thus $\mathcal{S}$ can be used to implement the role of $P_{2}^{a}$ in a split adversarial strategy). We also must relate to the fact that in the $\mathcal{F}_{\mathrm{BBCA}}$-hybrid model, $\mathcal{S}$ has full control over the functionality and so can modify values that are registered and so on. This is in contrast to $\mathcal{A}$ in a real execution that has no control over the functionality. Nevertheless, we will show that the operations of the functionality are so basic that this does not provide any real advantage to $\mathcal{S}$ (essentially, $\mathcal{Z}$ can verify that it is running in a world with a proper $\mathcal{F}_{\mathrm{BBCA}}$ functionality and so can prevent $\mathcal{S}$ from gaining any advantage). The proof works by considering three scenarios:

1. Scenario 1 - an $\mathcal{F}_{\mathrm{BBCA}}$-hybrid scenario with a corrupted $P_{1}$ : In this scenario we consider a specific real-world adversary $\mathcal{A}$ who has corrupted $P_{1}$, a specific environment $\mathcal{Z}$, and an execution of the protocol $\pi_{f}$ in the $\mathcal{F}_{\mathrm{BBCA}}$-hybrid model with an honest $P_{2}$.

2. Scenario 2 - an ideal-world scenario with a corrupted $P_{1}$ : In this scenario, we consider an ideal execution with the same $\mathcal{Z}$ and with the ideal-world adversary/simulator $\mathcal{S}$ that is guaranteed to exist for $\mathcal{A}$ and $\pi_{f}$. (Again here, $P_{1}$ is corrupted and $P_{2}$ is honest.)

3. Scenario 3-an $\mathcal{F}_{\mathrm{BBCA}}$-hybrid scenario with a corrupted $P_{2}$ : In this scenario we consider a new real adversary $\mathcal{A}^{\prime}$ who has corrupted $P_{2}$ and carries out a split adversarial strategy against an honest $P_{1}$.

The proof works by showing that certain properties hold in each of the scenarios. We proceed to the formal proof. Assume that the functionality $\mathcal{F}_{f}$ can be securely realized by a non-trivial protocol $\pi_{f}$. This implies that for every $\mathcal{F}_{\mathrm{BBCA}}$-hybrid adversary $\mathcal{A}$ there exist an ideal-process adversary/simulator $\mathcal{S}$ such that no environment $\mathcal{Z}$ can distinguish between an execution of the ideal process with $\mathcal{S}$ and $\mathcal{F}_{f}$ and an execution of the $\mathcal{F}_{\mathrm{BBCA}}$-hybrid protocol $\pi_{f}$ with $\mathcal{A}$. We begin by defining a specific $\mathcal{F}_{\mathrm{BBCA}}$-hybrid world scenario with a specific adversary $\mathcal{A}$ and environment $\mathcal{Z}$.

Scenario 1 - the first $\mathcal{F}_{\mathrm{BBCA}}$-hybrid scenario. The scenario consists of an environment $\mathcal{Z}$ and adversary $\mathcal{A}$, and honest parties $P_{1}$ and $P_{2}$. In this scenario, $P_{1}$ is corrupted (and thus controlled by $\mathcal{A}$ ) and $P_{2}$ is honest. Note that the protocol $\pi_{f}$ is run in the $\mathcal{F}_{\mathrm{BBCA}}$-hybrid model. This means that each party communicates with the ideal bulletin-board CA functionality during the execution of the protocol. We describe each entity's strategy separately:

- $\mathcal{A}$ 's strategy: $\mathcal{A}$ controls party $P_{1}$ who does nothing except to respond to specific requests of $\mathcal{A}$, as follows. When $P_{1}$ receives a message (register, sid, $v$ ) from $\mathcal{A}$, it sends the message (register, sid, $v$ ) to $\mathcal{F}_{\mathrm{BBCA}}$. (Note that $\mathcal{A}$ cannot send this message for $P_{1}$ and so $P_{1}$ must do it itself.) In addition, $\mathcal{A}$ mediates between $\mathcal{Z}$ and the $\mathcal{F}_{\mathrm{BBCA}}$ functionality. Specifically, when $\mathcal{Z}$ sends a message (register, sid, $v$ ) to $\mathcal{A}$, adversary $\mathcal{A}$ forwards this message to $P_{1}$ to forward to $\mathcal{F}_{\mathrm{BBCA}}$. Likewise, when $\mathcal{Z}$ sends a message (retrieve, sid, $P$ ) to $\mathcal{A}$ for some party $P$, adversary $\mathcal{A}$ forwards this message to the $\mathcal{F}_{\mathrm{BBCA}}$ functionality. When $\mathcal{A}$ receives the response $($ sid, $P, v)$ from $\mathcal{F}_{\mathrm{BBCA}}$ it forwards this response to $\mathcal{Z}$. Finally, $\mathcal{A}$ acts as a bridge between $\mathcal{Z}$ 
and $P_{2}$, delivering all messages from $\mathcal{Z}$ to $P_{2}$ (except for register and retrieve messages), and delivering all message from $P_{2}$ to $\mathcal{Z}$ (that $P_{2}$ sent to $P_{1}$ ).

- $\mathcal{Z}$ 's strategy: $\mathcal{Z}$ chooses inputs for the parties: $x_{1}$ for $P_{1}$ and $x_{2}$ for $P_{2}$, where $x_{2} \in_{R} X_{2}$ is randomly chosen, and $X_{2}$ is a polynomial-size set of inputs chosen by $\mathcal{Z}$. The environment $\mathcal{Z}$ writes $x_{2}$ on $P_{2}$ 's input tape and keeps $x_{1}$ to itself.

Next, $\mathcal{Z}$ plays the role of the honest $P_{1}$ on input $x_{1}$. That is, $\mathcal{Z}$ runs the honest $P_{1}$ 's protocol instructions on input $x_{1}$ and the incoming messages that it receives from $\mathcal{A}$. When instructed by the protocol $\pi_{f}$ to perform a key registration of some key $v, \mathcal{Z}$ sends (register, sid, $v$ ) to $\mathcal{A}$. When instructed by the protocol to retrieve a party $P$ 's key, $\mathcal{Z}$ sends (retrieve, sid, $P$ ) to $\mathcal{A}$. Upon receiving the answer $(\operatorname{sid}, P, v)$ from $\mathcal{A}$, it continues with the protocol and relates to the answer as if it was received directly from $\mathcal{F}_{\mathrm{BBCA}}$. (We stress that $\mathcal{Z}$ cannot interact directly with $\mathcal{F}_{\mathrm{BBCA}}$ in the UC model. Therefore, it must work through an intermediary.)

$\mathcal{Z}$ determines its output at the end of the protocol execution in the following way. First, it carries out consistency checks with respect to registered and retrieved keys. That is, $\mathcal{Z}$ outputs 0 if one of the following events occur during the execution:

1. $\mathcal{Z}$ asked to retrieve $P_{1}$ 's key but received a key that does not equal the one that $\mathcal{Z}$ registered previously (or a key is retrieved but $\mathcal{Z}$ never registered one).

2. $\mathcal{Z}$ asked to retrieve the key of some party $P$ more than once and received different keys in the different requests.

If the above checks pass, then $\mathcal{Z}$ looks at the local output that it received for $P_{1}$ and reads $P_{2}$ 's output tape. (Recall that $\mathcal{Z}$ plays $P_{1}$ so it receives some output from the protocol. We call this output the local- $P_{1}$ output.) $\mathcal{Z}$ outputs 1 if and only if the local- $P_{1}$ output equals $f_{1}\left(x_{1}, x_{2}\right)$ and $P_{2}$ 's output equals $f_{2}\left(x_{1}, x_{2}\right)$.

We have the following claim:

Claim 3.2 In the $\mathcal{F}_{\mathrm{BBCA}}$-hybrid world described in scenario number 1 , the environment $\mathcal{Z}$ outputs 1 with probability that is negligibly close to 1 .

Proof: Observe that an execution of $\pi_{f}$ with the above $\mathcal{Z}$ and $\mathcal{A}$ looks exactly like an execution between two honest parties $P_{1}$ and $P_{2}$ upon inputs $x_{1}$ and $x_{2}$, respectively. This is due to the fact that $\mathcal{Z}$ plays the honest $P_{1}$ and the adversary $\mathcal{A}$ honestly relays all messages between $\mathcal{Z}$ and $P_{2}$ and $\mathcal{Z}$ and $\mathcal{F}_{\mathrm{BBCA}}$ (where these latter messages are sent via $P_{1}$ because only it can register keys for its identity). It follows that since $\pi_{f}$ is a non-trivial protocol, both parties receive output. In the case of honest $P_{1}$ and $P_{2}$ these outputs are $f_{1}\left(x_{1}, x_{2}\right)$ and $f_{2}\left(x_{1}, x_{2}\right)$ respectively, except with negligible probability (otherwise, an environment would be able to distinguish the real and ideal models when no party is corrupted). Since all consistency checks by $\mathcal{Z}$ pass in this scenario (because $\mathcal{A}$ always "behaves well"), it follows that $\mathcal{Z}$ outputs 1 except with negligible probability.

Scenario 2 - the ideal world with $\mathcal{F}_{f}$. In this scenario, we have the same environment $\mathcal{Z}$ as above, dummy parties $P_{1}$ and $P_{2}$ (where $P_{1}$ is corrupted), and the ideal adversary/simulator that is guaranteed to exist by the security of the protocol $\pi_{f}$; denote this adversary by $\mathcal{S}$. The strategy of $\mathcal{S}$ is not determined by us. Nevertheless we prove that the following properties must hold: 
1. Property 1: If $\mathcal{Z}$ sends a message (retrieve, sid, $P_{1}$ ) to $\mathcal{S}$ and it previously sent a (register, sid, $v$ ) message, then the value $v_{s}$ in $\mathcal{S}$ 's response equals $v$. Otherwise, if no register message was sent, it holds that $v_{s}=\perp$.

2. Property 2: If $\mathcal{Z}$ sends a message (retrieve, sid, $P_{2}$ ) to $\mathcal{S}$ and it has previously received a response $\left(\operatorname{sid}, P_{2}, v\right)$ for $v \neq \perp$, then the value $v_{s}$ in $\mathcal{S}$ 's response equals $v$.

The fact that these properties hold follow from the inspection of $\mathcal{Z}$ 's consistency checks. Specifically, if with non-negligible probability one of the properties does not hold, then $\mathcal{Z}$ will output 0 with nonnegligible probability in the ideal execution. Since $\mathcal{Z}$ outputs 1 except with negligible probability in the $\mathcal{F}_{\mathrm{BBCA}}$-hybrid execution, this implies that $\mathcal{Z}$ distinguishes the $\mathcal{F}_{\mathrm{BBCA}}$-hybrid and ideal executions, in contradiction to the security of the protocol. (The above essentially proves that although $\mathcal{S}$ plays $\mathcal{F}_{\mathrm{BBCA}}$ in this execution and so can theoretically do anything it wishes, it is actually very limited in what it can do.) We are now ready to prove the following claim.

Claim 3.3 In the ideal execution, $\mathcal{S}$ must send $\mathcal{F}_{f}$ an input $x_{1}^{\prime}$ for which it holds that for every $x_{2} \in X_{2}, f_{2}\left(x_{1}, x_{2}\right)=f_{2}\left(x_{1}^{\prime}, x_{2}\right)$, except with negligible probability. Furthermore, $\mathcal{Z}$ 's local- $P_{1}$ output equals $f_{1}\left(x_{1}, x_{2}\right)$ except with negligible probability.

Proof: Assume by contradiction that with non-negligible probability $\mathcal{S}$ sends an input $x_{1}^{\prime}$ to $\mathcal{F}_{f}$ such that for some $\tilde{x}_{2} \in X_{2}$ it holds that $f_{2}\left(x_{1}^{\prime}, \tilde{x}_{2}\right) \neq f_{2}\left(x_{1}, \tilde{x}_{2}\right)$. This implies that if $P_{2}$ has input $\tilde{x}_{2}$, it will output $f_{2}\left(x_{1}^{\prime}, \tilde{x}_{2}\right) \neq f_{2}\left(x_{1}, \tilde{x}_{2}\right)$. By the specification of $\mathcal{Z}$, when this occurs $\mathcal{Z}$ outputs 0 . In order to analyze the probability that $P_{2}$ 's input equals $\tilde{x}_{2}$, recall that $X_{2}$ is of polynomial size and $P_{2}$ 's input is uniformly chosen from $X_{2}$. Furthermore, the probability that $\mathcal{S}$ sends this "bad" $x_{1}^{\prime}$ is independent of the choice of $x_{2}$ for $P_{2}$ (because $\mathcal{S}$ has no information about $x_{2}$ when it sends $x_{1}^{\prime}$ ). Therefore, the probability that $\mathcal{Z}$ outputs 0 is at least $1 /\left|X_{2}\right|$ times the probability that $\mathcal{S}$ outputs the bad $x_{1}^{\prime}$ (which we have already said is non-negligible). Thus, $\mathcal{Z}$ outputs 0 in the ideal process with non-negligible probability. This contradicts the security of $\pi_{f}$ because as we have already seen, $\mathcal{Z}$ outputs 0 in an $\mathcal{F}_{\mathrm{BBCA}}$-hybrid execution with at most negligible probability.

Regarding the "furthermore" part of the claim, this follows directly from the fact that if $\mathcal{Z}$ 's local- $P_{1}$ output does not equal $f_{1}\left(x_{1}, x_{2}\right)$ then it outputs 0 .

We remark that the above proof does not use the properties that we proved regarding $\mathcal{S}$ and the register/retrieve messages. These properties will be used below.

Scenario 3 - the second $\mathcal{F}_{\mathrm{BBCA}}$-hybrid scenario. As with the first scenario, here we also consider an $\mathcal{F}_{\mathrm{BBCA}}$-hybrid execution of $\pi_{f}$. However, here $P_{1}$ is honest while $P_{2}$ is corrupted (in contrast to scenario 1). We describe a split adversarial strategy for $P_{2}$ that uses $\mathcal{S}$ in this scenario, and then show that it is successful. We begin by describing $P_{2}^{a}$ (we will not refer to an adversary $\mathcal{A}^{\prime}$ since $\left(P_{2}^{a}, P_{2}^{b}\right)$ is the adversary here). Machine $P_{2}^{a}$ internally invokes the simulator $\mathcal{S}$ and emulates an ideal process execution of $\mathcal{S}$ with $\mathcal{F}_{f}$ and the above $\mathcal{Z}$, while actually running an $\mathcal{F}_{\text {BBCA-hybrid }}$ execution of $\pi_{f}$ with $P_{1}$. It works as follows:

1. When $P_{1}$ sends (register, sid, $v$ ) to $\mathcal{F}_{\mathrm{BBCA}}$, machine $P_{2}^{a}$ delivers the message to $\mathcal{F}_{\mathrm{BBCA}}$ and internally hands the message to $\mathcal{S}$ as if it was received from $\mathcal{Z}$. (Recall that the ideal messages sent between the honest parties and ideal functionalities are sent via the adversary, and so $P_{2}^{a}$ receives these messages.) When $P_{1}$ sends a (retrieve, sid, $P_{1}$ ) request to $\mathcal{F}_{\mathrm{BBCA}}, P_{2}^{a}$ hands it to $\mathcal{S}$ and delivers the response when $\mathcal{S}$ sends the message $\left(\right.$ sid $\left., P_{1}, v\right)$ that is intended as a reply to $\mathcal{Z}$. 
2. When $P_{1}$ sends a (retrieve, sid, $P_{2}$ ) to $\mathcal{F}_{\mathrm{BBCA}}, P_{2}^{a}$ internally hands this message to $\mathcal{S}$, as if $\mathcal{S}$ received it from $\mathcal{Z}$. When $\mathcal{S}$ outputs the response $\left(\right.$ sid, $\left.P_{2}, v_{s}\right), P_{2}^{a}$ checks if there is a recorded tuple ( $\left.\operatorname{sid}, P_{2}, v\right)$ where $v=v_{s}$ (i.e., $P_{2}^{a}$ checks if $v_{s}$ was already registered for this $s i d$ ). If no such tuple exists $P_{2}^{a}$ sends the message (register, sid, $v_{s}$ ) to $\mathcal{F}_{\mathrm{BBCA}}$, and only then delivers the retrieve request of $P_{1}$. (In addition, $P_{2}^{a}$ records the tuple (sid, $\left.P_{2}, v_{s}\right)$ internally.)

3. Every message that $P_{2}^{a}$ receives from the honest $P_{1}$ in the execution of $\pi_{f}$, it forwards to $\mathcal{S}$ as if $\mathcal{S}$ received it from $\mathcal{Z}$.

4. Every message (other than responses to retrieve requests) that $\mathcal{S}$ sends to $\mathcal{Z}$ in the emulation, $P_{2}^{a}$ forwards to $P_{1}$ in the real execution.

5. When $\mathcal{S}$ outputs a value $x_{1}^{\prime}$ that it intends to send to $\mathcal{F}_{f}$, entity $P_{2}^{a}$ hands it to $P_{2}^{b}$. Then, when $P_{2}^{b}$ hands it back a value $y_{1}^{\prime}$ it passes this to $\mathcal{S}$ as if it was received from $\mathcal{F}_{f}$ and continues the emulation as above. (Recall that $y_{1}^{\prime}$ is computed by $P_{2}^{b}$ choosing some $x_{2}^{\prime} \in X_{2}$ of its choice and then computing $y_{1}^{\prime}=f_{1}\left(x_{1}^{\prime}, x_{2}^{\prime}\right)$; see Definition 2.2.)

We now show that the distribution of messages received and sent by $\mathcal{S}$ and $P_{1}$ in this $\mathcal{F}_{\mathrm{BBCA}^{-}}$ hybrid execution with $P_{2}=\left(P_{2}^{a}, P_{2}^{b}\right)$ is statistically close to the distribution of messages received and sent by $\mathcal{S}$ and $\mathcal{Z}$ in scenario 2 . We focus first on the messages that relate to the $\mathcal{F}_{\mathrm{BBCA}}$ functionality:

1. When $P_{1}$ sends a (register, sid, $v$ ) message to $\mathcal{F}_{\mathrm{BBCA}}$, machine $P_{2}^{a}$ gives the message to $\mathcal{S}$ before actually forwarding it to the functionality. Likewise, in scenario $2, \mathcal{S}$ receives the same message from $\mathcal{Z}$.

2. When $P_{1}$ sends a (retrieve, sid, $P_{2}$ ) message to $\mathcal{F}_{\mathrm{BBCA}}$, machine $P_{2}^{a}$ first gives the message to $\mathcal{S}$, just as in scenario 2 . Furthermore, in scenario $3, P_{1}$ receives $\left(\right.$ sid, $\left.P_{2}, v_{s}\right)$ in response from $\mathcal{F}_{\mathrm{BBCA}}$, where $v_{s}$ is chosen by $\mathcal{S}$. This is exactly the same as what happens in scenario 2 .

3. When $P_{1}$ sends duplicate retrieve messages with the same sid in scenario $3, P_{2}^{a}$ does not perform another registration of $v_{s}$ since the value is already registered inside $\mathcal{F}_{\mathrm{BBCA}}$. As a result, $P_{1}$ receives the same $v_{s}$ from $\mathcal{F}_{\mathrm{BBCA}}$ in both requests. Nevertheless, by property 2 described above in scenario $2, \mathcal{S}$ returns the same value in scenario 2 (except with negligible probability).

4. When $P_{1}$ sends (retrieve, sid, $P_{1}$ ) to $\mathcal{F}_{\mathrm{BBCA}}$ in scenario $3, \mathcal{S}$ receives this message from $P_{2}^{a}$. In scenario $2, \mathcal{S}$ receives the same message from $\mathcal{Z}$. Now, in scenario $3, P_{1}$ receives the retrieved value from $\mathcal{F}_{\mathrm{BBCA}}$ and $\mathcal{S}$ cannot modify its value (this value was previously registered by $\left.P_{1}\right)$. Nevertheless, by property 1 described above in scenario $2, \mathcal{S}$ returns the same value in scenario 2 (except with negligible probability).

Finally, we observe that since $\mathcal{Z}$ plays the honest $P_{1}$ strategy with input $x_{1}$, the distribution over the messages that it interchanges with $\mathcal{S}$ in scenario 2 is statistically close to the distribution over the messages that $P_{1}$ interchanges with $\mathcal{S}$ via $P_{2}^{a}$ in scenario 3 (the only difference is due to the negligible probability that properties 1 and 2 may not hold). We conclude that up until the point that $P_{2}^{a}$ hands $x_{1}^{\prime}$ to $P_{2}^{b}$, the distributions in the two scenarios are statistically close. Therefore, the distribution over the value $x_{1}^{\prime}$ that $P_{2}^{a}$ hands to $P_{2}^{b}$ in scenario 3 is statistically close to the distribution over the value $x_{1}^{\prime}$ that $\mathcal{S}$ sends to $\mathcal{F}_{f}$ in scenario 2 . This implies that $P_{2}=\left(P_{2}^{a}, P_{2}^{b}\right)$ is a successful split adversarial strategy with respect to requirement (1) of Definition 2.3 (see Section 2.2). 
It remains to show that $P_{2}$ is also a successful split adversarial strategy with respect to requirement (2) of Definition 2.3. This can be shown in exactly the same way as in [12]. Namely, by Claim 3.3 we have that $\mathcal{Z}$ 's local- $P_{1}$ output must equal $f_{1}\left(x_{1}, x_{2}\right)$ except with negligible probability. Now, assume by contradiction that with non-negligible probability $P_{1}$ outputs a value $\tilde{y}_{1}$ that does not equal $f_{1}\left(x_{1}, x_{2}^{\prime}\right)$ in scenario 3 . Recall that $P_{2}^{b}$ hands $P_{2}^{a}$ the value $y_{1}^{\prime}$ that is computed by first choosing $x_{2}^{\prime}$ following an arbitrary (polynomial-time) strategy of its choice, and then computing $y_{1}^{\prime}=f_{1}\left(x_{1}^{\prime}, x_{2}^{\prime}\right)$. Now, modify $P_{2}^{b}$ to a machine $\tilde{P}_{2}^{b}$ who chooses $x_{2}^{\prime}$ uniformly from $X_{2}$. Since $X_{2}$ is of polynomial-size, it follows that with probability $1 / \operatorname{poly}(n)$, the value $x_{2}^{\prime}$ chosen by $\tilde{P}_{2}^{b}$ equals that chosen by $P_{2}^{b}$. Thus, if $P_{1}$ outputs $\tilde{y}_{1} \neq f_{1}\left(x_{1}, x_{2}^{\prime}\right)$ with non-negligible probability with $P_{2}^{b}$ then it will also output $\tilde{y}_{1} \neq f_{1}\left(x_{1}, x_{2}^{\prime}\right)$ with non-negligible probability in the modified scenario with $\tilde{P}_{2}^{b}$. However, now notice that in this modified scenario, the value $x_{2}^{\prime}$ is chosen in exactly the same way as $\mathcal{Z}$ chooses it in scenario 2 (namely, it is chosen uniformly from $X_{2}$ ). Furthermore, the value $y_{1}^{\prime}$ handed to $P_{2}^{a}$ by $P_{2}^{b}$ in scenario 3 is distributed exactly like the value that $\mathcal{S}$ receives from $\mathcal{F}_{f}$ in scenario 2 (because the ideal functionality received $x_{1}^{\prime}$ from $\mathcal{S}$ and $x_{2}$ from the honest $P_{2}$ and $x_{2}$ is distributed exactly like $x_{2}^{\prime}$ ). Now, as we have mentioned, $\mathcal{Z}$ outputs 0 if its local- $P_{1}$ output is different from $f_{1}\left(x_{1}, x_{2}\right)$ (which has exactly the same distribution as $f_{1}\left(x_{1}, x_{2}^{\prime}\right)$ ). We therefore conclude that $P_{1}$ must also output $f_{1}\left(x_{1}, x_{2}^{\prime}\right)$ in scenario 3 , except with negligible probability (here we again apply the fact that the distribution over all messages seen in the two scenarios are statistically close). Thus, $P_{2}=\left(P_{2}^{a}, P_{2}^{b}\right)$ is also a successful split adversarial strategy with respect to requirement (2) of Definition 2.3. This completes the proof of the Lemma 3.1.

Extension to unique keys. The above proof can be extended in a straightforward way to the case that the bulletin-board functionality $\mathcal{F}_{\mathrm{BBCA}}$ ensures that all public keys are unique. This is due to the fact that after $\mathcal{Z}$ registers its own key it can retrieve $P_{2}$ 's key and check that it is different from its own. Thus, such a strengthening of $\mathcal{F}_{\mathrm{BBCA}}$ is not helpful for constructing UC secure protocols.

\subsection{Bulletin-Board CA with Independent Keys}

We now further strengthen the public-key model and require that all the public keys be independent of one another. This is achieved by requiring that all parties complete registration before any retrieval requests are made. Of course, we also require that the adversary not see the public-keys before all registration has finished. Technically, this is achieved by defining the public-key to be part of the "private contents" of the messages sent between the honest parties and ideal functionality. The functionality is denoted $\mathcal{F}_{\text {INDCA }}$ (for independent-key CA) and is defined in Figure 2.

We have the following theorem:

Theorem 3.4 Assume that enhanced trapdoor permutations and dense cryptosystems exist. Then, for any multi-party ideal functionality $\mathcal{F}$, there exists a non-trivial protocol $\pi$ that UC realizes $\mathcal{F}$ in the $\mathcal{F}_{\mathrm{INDCA}}$-hybrid model in the presence of malicious, static adversaries, and for any number of corruptions.

The theorem is proven by showing how the common reference string functionality $\mathcal{F}_{\text {CRS }}$ with a uniformly distributed string can be UC realized in the $\mathcal{F}_{\text {INDCA}}$-hybrid model. We then apply the results of [14] that show that any functionality can be UC realized in the $\mathcal{F}_{\mathrm{CRS}}$-hybrid model. (We remark that the above theorem can also be stated for adaptive adversaries. We state the static version for the sake of simplicity.) 


\section{Functionality $\mathcal{F}_{\text {INDCA }}$}

$\mathcal{F}_{\text {INDCA }}$ initializes a variable allow to 1 and proceeds as follows, running with parties $P_{1}, P_{2}, \ldots$ and an adversary $\mathcal{S}$ :

- Register commands: Upon receiving a message (register, sid, $v$ ) from some party $P_{i}$, the functionality checks that allow $=1$ and that $P_{i}$ has not already registered a key $v^{\prime}$. If the checks pass, then it records the pair $\left(P_{i}, v\right)$. Otherwise, it ignores the new message. (The public-header of the register command consists of (register, sid) and the private contents consists of $v$.)

- Retrieve commands: Upon receiving a message (retrieve, sid, $P_{i}$ ) from some party $P_{j}$ or the adversary $\mathcal{S}$, the functionality checks if some pair $\left(P_{i}, v\right)$ is recorded. If yes, and $v$ is the recorded value, it sets allow $=0$ and sends $\left(\operatorname{sid}, P_{i}, v\right)$ to $P_{j}$ (or $\left.\mathcal{S}\right)$. Otherwise, it returns $\left(\operatorname{sid}, P_{i}, \perp\right)$. (The entire retrieve command can be placed in the public header.)

Figure 2: The Independent-Key CA functionality

\section{Functionality $\mathcal{F}_{\mathrm{CRS}}$}

$\mathcal{F}_{\text {CRS }}$ proceeds as follows, running with parties $P_{1}, P_{2}, \ldots$ and an adversary $\mathcal{S}$ :

- Upon receiving a message (crs, sid) from some party $P_{i}$, the functionality checks if a pair $($ sid, $r)$ has been recorded. If yes, it returns $(\mathrm{crs}, s i d, r)$ to $P_{i}$. Otherwise, it chooses a uniformly distributed $r$ (of a given length), records $\left(\right.$ sid, $r$ ) and returns (crs, sid, $r$ ) to $P_{i}$.

Figure 3: The uniformly distributed common reference string functionality

For the sake of completeness, we present the $\mathcal{F}_{\mathrm{CRS}}$ functionality in Figure 3. We note that the functionality is fixed with a given polynomial, mandating the length of the reference string. We prove the following:

Claim 3.5 The $\mathcal{F}_{\mathrm{CRS}}$ functionality can be $U C$ realized in the $\mathcal{F}_{\mathrm{INDCA}}$-hybrid model, for any number of corrupted parties.

Proof: The proof of this claim is straightforward and follows from the simple observation that in the $\mathcal{F}_{\text {INDCA }}$-hybrid model it is possible to carry out perfect coin-tossing. Namely, all parties register uniformly distributed strings (of a given length). Once a party has registered its string, it sends a message saying that it has done so to all others. When a party receives messages from all others that they have registered, it retrieves all the strings and defines the common reference string to be the exclusive-or of all the strings. (We stress that the party checks that all parties have indeed registered keys. If not, it aborts.) Clearly the result is uniformly distributed, because the adversary does not know any of the honest parties' registered strings when the corrupted parties register their strings.

We conclude by commenting on where the proof of Lemma 3.1 fails in this setting. This can be seen by noting that in scenario $2, \mathcal{Z}$ must register its public-key via $\mathcal{S}$. Thus, $\mathcal{S}$ essentially learns $P_{1}$ 's key before it needs to register its own. This is in contrast to scenario 3 where the key registered by $P_{1}$ is secret until $P_{2}$ registers its own key. 


\subsection{The Bare Public-Key Model}

The bare public-key model, as introduced by [11], has the property that parties can only register public keys before the protocol executions begin. Typically, this model has been considered for concurrent self composition where the secure protocol is the only protocol running. There are two ways of interpreting this in the setting of concurrent general composition. An interpretation leading to a more realistic model is one that states that the only limitation is that the public keys are registered before the secure protocol begins. In particular, other protocols may be running during the key registration phase. (Stated differently, the requirement that the public keys all be registered before the secure protocol begins is one that is local to the secure protocol, and is not a global network requirement.) We remark that this model is very realistic because it is possible to set a date where the secure protocol begins running and to close key registration before this time. A second interpretation is that no protocols may run during the key registration phase. We view this as highly unrealistic, but nevertheless study it in Section 3.4.

We remark that the bare public-key model has been used in a number of papers in order to bypass lower bounds and impossibility results. For example, it was used by [11] (and many later works) in order to construct constant-round resettable zero-knowledge protocols (something that is impossible in the plain model). Recently, it has been used to achieve stronger notions of concurrent non-malleable zero-knowledge $[18,29] .{ }^{5}$ Our results here show that it is impossible to further strengthen these results to achieve protocols that are UC-secure (or equivalently, secure under concurrent general composition).

We define the bare-public key functionality in Figure 4. The functionality has an internal Boolean flag, called allow, which is set to 0 once one of the parties sends the message HaltRegistration. This step represents the end of the registration phase and from this point on, the functionality rejects any registration request from any party. We allow any party to call HaltRegistration to ensure that no honest party begins running the secure protocol before the registration phase is halted. We stress that as with all the CA functionalities we have seen so far, with the exception of $\mathcal{F}_{\text {INDCA }}$, the public keys are not secret during the registration phase. (If this were the case, then $\mathcal{F}_{\mathrm{BPK}}$ would just be a special case of $\mathcal{F}_{\text {INDCA }}$.)

\section{Functionality $\mathcal{F}_{\mathrm{BPK}}$}

$\mathcal{F}_{\mathrm{BPK}}$ initializes a variable allow to 1 and proceeds as follows, running with parties $P_{1}, P_{2}, \ldots$ and an adversary $\mathcal{S}$ :

- Register commands: Upon receiving a message (register, sid, $v$ ) from some party $P_{i}$, the functionality checks that allow $=1$ and that $P_{i}$ has not already registered a key $v^{\prime}$. If the checks pass, then it records the pair $\left(P_{i}, v\right)$. Otherwise, it ignores the new message.

- Retrieve commands: Upon receiving a message (retrieve, sid, $P_{i}$ ) from some party $P_{j}$ or the adversary $\mathcal{S}$, the functionality checks if some pair $\left(P_{i}, v\right)$ is recorded. If yes, and $v$ is the recorded value, it sends $\left(\operatorname{sid}, P_{i}, v\right)$ to $P_{j}($ or $\mathcal{S})$. Otherwise, it returns $\left(\operatorname{sid}, P_{i}, \perp\right)$.

- Halt registration commands: Upon receiving a message (HaltRegistration, sid) from any party $P_{i}$ or the adversary $\mathcal{S}$, the functionality sets allow $=0$.

Figure 4: The bare public-key model functionality

\footnotetext{
${ }^{5}$ We remark that [18] call their model the "authenticated public-key model". Nevertheless, this is the same as the bare public-key model that was considered previously and is considered here.
} 
We have the following lemma:

Lemma 3.6 Let $f$ be a polynomial-time two-party function, and let $\mathcal{F}_{f}$ be the two-party ideal functionality that receives $x_{1}$ from $P_{1}$ and $x_{2}$ from $P_{2}$, and hands them back their respective outputs $f_{1}\left(x_{1}, x_{2}\right)$ and $f_{2}\left(x_{1}, x_{2}\right)$. If $\mathcal{F}_{f}$ can be $U C$-realized in the $\mathcal{F}_{\mathrm{BPK}}$-hybrid model by a non-trivial protocol $\pi_{f}$, then there exists a machine $P_{2}^{a}$ such that for every machine $P_{2}^{b}$ of the form described in Definition 2.2, the split adversarial strategy for $P_{2}=\left(P_{2}^{a}, P_{2}^{b}\right)$ is successful, except with negligible probability.

Proof: Without loss of generality, we focus on canonical secure protocols that begin with the following steps (the instructions are stated for party $P_{i}$ and are the same for all parties):

1. Party $P_{i}$ chooses a public-key $v_{i}$ (according to the protocol instructions) and sends (register, sid, $v_{i}$ ) to $\mathcal{F}_{\mathrm{BPK}}$.

2. For each $j \neq i$, party $P_{i}$ sends (retrieve, sid, $P_{j}$ ) to $\mathcal{F}_{\mathrm{BPK}}$ and saves the retrieved key. If it receives back a tuple $\left(\right.$ sid $\left., P_{j}, \perp\right)$, as would be the case if $P_{j}$ has not yet registered a key, then it continues trying to retrieve the key.

3. After retrieving all the parties' keys, $P_{i}$ sends (HaltRegistration, sid) and begins the secure protocol execution.

4. Whenever $P_{i}$ is instructed to retrieve a key within the secure protocol, it takes the appropriate key that was previously stored.

Our focus on canonical protocols is without loss of generality because under the assumption that all key registration takes place before the protocol executions begin, nothing is lost by having the parties retrieve all keys before the protocol begins.

Once we are given that the secure protocol is as above, the proof of the lemma here is almost identical to that Lemma 3.1. The only important observation relates to scenario 3. Recall that the corrupted $P_{2}$ does not run the canonical protocol described above, but rather works as in Lemma 3.1. In particular, the strategy of machine $P_{2}^{a}$ within $P_{2}$ is such that when $P_{1}$ first asks to retrieve $P_{2}$ 's key, $P_{2}^{a}$ sends this request to $\mathcal{S}$ who returns a key $v_{s}$. Party $P_{2}$ registers this key $v_{s}$ with the CA functionality and only then delivers the retrieve request from $P_{1}$ to the functionality. In principle, this is a problem in the bare public-key model because the key $v_{s}$ is chosen later (and may depend on the protocol messages). Nevertheless, observe that this registration of $v_{s}$ only takes place in the first retrieve request. In contrast, in all later retrieve requests $P_{2}$ does nothing but deliver the retrieve requests and responses between $P_{1}$ and the functionality. Now, in the canonical protocol form, $P_{1}$ retrieves $P_{2}$ 's key before sending a HaltRegistration message. This ensures that the first retrieval of $P_{2}$ 's key is carried out during the registration phase, and thus $P_{2}^{a}$ can still register the key $v_{s}$ that $\mathcal{S}$ chooses.

\subsection{A Strong Bare Public-Key Model}

As we have mentioned, it is possible to consider an even stronger bare public-key model in which it is guaranteed that no protocols whatsoever are executing during the key registration phase. This can be formally modeled in the UC framework by having a period where $\mathcal{Z}$ cannot interact with $\mathcal{A}$. In this case, it is easy to see that UC security can be achieved by just having all parties run a single coin-tossing protocol that is secure in the stand-alone model (since no other protocols are 
running, the protocol runs "stand-alone"). The result of the coin-tossing is then taken as a common reference string, thereby allowing the use of [14] to UC-realize any functionality. We personally do not find this setting very interesting because it seems highly unrealistic to expect that there be a sterile period where no protocols whatsoever are executed.

Of course, the proof of Lemma 3.1 in this case is due to the fact that $\mathcal{Z}$ cannot register the public key in scenarios 1 and 2. Thus, the key must be chosen by $\mathcal{A}$ (or $\mathcal{S}$ ), giving $\mathcal{S}$ an advantage.

\subsection{Active Key Registration by the CA}

All of the public-key models that we have seen above have the property that the CA is passive in that it merely accepts keys (albeit while invoking certain checks). In this section, we consider the key registration functionality introduced by [2] that plays a more active role.

\section{Functionality $\mathcal{F}_{\mathrm{KR}}^{f}$}

$\mathcal{F}_{\mathrm{KR}}^{f}$ is parameterized by a function $f$ and a security parameter $n$. It initializes a set $R$ of strings to be empty at the first activation and then proceeds as follows, running with parties $P_{1}, P_{2}, \ldots$ and an adversary $\mathcal{S}$ :

- Register commands: Upon receiving a message (register, sid) from some party $P_{i}$ (that is either corrupted or uncorrupted), the functionality sends (register, sid, $P_{i}$ ) to $\mathcal{S}$ and receives a value $p^{\prime}$ back. Then, if $p^{\prime} \in R$, it sets $p=p^{\prime}$. Otherwise, it chooses a random string $r \in_{R}\{0,1\}^{n}$, sets $p=f(r)$ and adds $p$ to $R$. Finally, it records the pair $\left(P_{i}, f(r)\right)$ and sends (registered, sid, $p$ ) to $P_{i}$ and $\mathcal{S}$.

- Register by a corrupted party: Upon receiving a message (register, sid, $r$ ) from a corrupted party $P_{i}$, the functionality records $\left(P_{i}, f(r)\right)$. In this case, $f(r)$ is not added to $R$.

- Retrieve commands: Upon receiving a message (retrieve, sid, $P_{i}$ ) from some party $P_{j}$, the functionality sends a message (retrieve, sid, $\left.P_{i}, P_{j}\right)$ to $\mathcal{S}$ and obtains a value $p$ back from $\mathcal{S}$. If $\left(P_{i}, p\right)$ is recorded, then the functionality returns $\left(\operatorname{sid}, P_{i}, p\right)$ to $P_{j}$. Else, it returns $\left(\operatorname{sid}, P_{i}, \perp\right)$ to $P_{j}$.

Figure 5: The key registration functionality of [2]

In [2] it has been shown that essentially any functionality can be UC-realized in the $\mathcal{F}_{\mathrm{KR}}^{f}$-hybrid model, for any number of corrupted parties. Thus, clearly an analogue to Lemma 3.1 cannot be proven for this model. Our aim in this section is therefore to explain where the proof of Lemma 3.1 fails when considering the $\mathcal{F}_{\mathrm{KR}}^{f}$ functionality. This highlights the crucial property of the $\mathcal{F}_{\text {KR }}^{f}$ functionality that enables the construction of protocols and thus can be useful when attempting to design other CA functionalities that can be used for constructing UC-secure protocols.

Failure of Lemma 3.1 in the $\mathcal{F}_{\mathrm{KR}}^{f}$-hybrid model. In the second scenario of Lemma 3.1 the simulator $\mathcal{S}$ plays the role of the CA functionality $\mathcal{F}_{\mathrm{KR}}^{f}$. In particular, this means that it determines the value $r$ to be used in computing a new key $f(r)$. The main issue that arises here is that, depending on the choice of the function $f$, it may not be possible for $\mathcal{Z}$ to distinguish the case that a key $p$ is correctly formed (i.e., by choosing $r$ and computing $f(r)$ ) from the case that is not correctly formed. Indeed, in the construction by [2], they utilize the fact that keys can be generated in alternate ways that all look indistinguishable from the defined generation $f(r)$. Of course, this is crucial when moving to scenario 3 , because party $P_{2}$ cannot choose the keys as it wishes and so cannot emulate scenario 2 while in scenario 3 . The conclusion is that in order 
to overcome these impossibility results, a CA functionality is needed that breaks the symmetry between the capabilities of the ideal simulator $\mathcal{S}$ when "playing the role" of the functionality and a true corrupted party who interacts with the actual functionality.

\subsection{CA Corruptions and UC Security}

In this section we investigate the possibility of obtaining UC secure protocols even when the CA may be corrupted. Of course, this is of interest only for public-key models for which it is possible to achieve UC security (without corruptions). Thus, for example, this is of interest in the $\mathcal{F}_{\mathrm{CRS}}$, $\mathcal{F}_{\mathrm{KR}}^{f}$ and $\mathcal{F}_{\text {INDCA }}$ models. We also note that if full malicious corruptions are allowed, then there is no difference between the public-key model and the plain model (the functionality can be viewed as a regular party). Thus, UC security can clearly not be realized in such a case. We therefore consider weaker corruptions. We will state out results for general CA functionalities and not specific ones; see below.

\subsubsection{Passive (Semi-Honest) CA Corruptions}

We say that a CA functionality is passively corrupted if the adversary has access to the internal state of the functionality, but the functionality continues to act according to its specification. (Thus, the adversary has malicious corruptions of regular parties but only semi-honest corruptions of the CA functionality). We show that there exists a CA functionality that enables the construction of UC secure protocols even under passive corruptions. The functionality is the common-reference string functionality $\mathcal{F}_{\text {CRS }}$ defined with a uniformly distributed string, as in Figure 3 . We have the following theorem:

Theorem 3.7 Assume that enhanced trapdoor permutations and dense cryptosystems exist. Then, for any multi-party ideal functionality $\mathcal{F}$, there exists a non-trivial protocol $\pi$ that UC realizes $\mathcal{F}$ in the $\mathcal{F}_{\mathrm{CRS}}$-hybrid model in the presence of malicious, static adversaries, and for any number of corruptions. This holds even when the $\mathcal{F}_{\mathrm{CRS}}$ functionality is run by a party that is corrupted in a semi-honest fashion.

The proof of the theorem follows from the fact that UC secure protocols can be achieved in the uniformly distributed $\mathcal{F}_{\mathrm{CRS}}$, as shown in [14]. This is due to the fact that when the common reference string is uniformly distributed, the functionality has no additional internal state. Thus, the semi-honest corruption provides no advantage to the adversary.

There is one important point to note regarding the above. Namely, in order to prove security in this model (where the adversary has access to the internal state of $\mathcal{F}_{\text {CRS }}$ ) it must be the case that the common reference string is generated truly randomly and not pseudorandomly. Formally, this is achieved by having $\mathcal{F}_{\mathrm{CRS}}$ simply output random bits taken directly from its random tape. This is needed because the UC simulator must be able to choose the random tape as it wishes (typically so that it includes some type of trapdoor), and if $\mathcal{F}_{\mathrm{CRS}}$ takes a smaller part of its random tape and applies a pseudorandom generator, the simulator will not be able to do this. Note that in practice, this means that a (semi) trusted party who provides the $\mathcal{F}_{\mathrm{CRS}}$ service must generate the common reference string using a pure hardware method. In particular, it cannot take a small random seed and apply a pseudorandom generator. 


\subsubsection{Indistinguishable Malicious CA Corruptions}

An indistinguishable malicious corruption of the CA entity means that the adversary has full control over the CA, with the limitation that the distribution over the messages produced by the corrupted $\mathrm{CA}$ is indistinguishable from the distribution over the messages produced by an honest CA. Stated differently, the CA behaves in an honest-looking manner; see [15]. ${ }^{6}$ We show that Lemma 3.1 holds also in this scenario.

Lemma 3.8 Let $f$ be a polynomial-time two-party function, and let $\mathcal{F}_{f}$ be the two-party ideal functionality that receives $x_{1}$ from $P_{1}$ and $x_{2}$ from $P_{2}$, and hands them back their respective outputs $f_{1}\left(x_{1}, x_{2}\right)$ and $f_{2}\left(x_{1}, x_{2}\right)$. If $\mathcal{F}_{f}$ can be $U C$-realized in the $\mathcal{F}_{\mathrm{BBCA}}$-hybrid model by a non-trivial protocol $\pi_{f}$, then there exists a machine $P_{2}^{a}$ such that for every machine $P_{2}^{b}$ of the form described in Definition 2.2, the split adversarial strategy for $P_{2}=\left(P_{2}^{a}, P_{2}^{b}\right)$ is successful, except with negligible probability. This holds even if $\mathcal{F}_{\mathrm{BBCA}}$ is only honest-looking, as defined in [15].

Proof: Once again, the proof is very similar to the proof of Lemma 3.1. Recall that in scenario 3 , party $P_{2}$ is corrupted and internally runs the code of the simulator $\mathcal{S}$ that is guaranteed to exist in scenario 2 . In scenario $3, P_{2}$ emulates the ideal process with $\mathcal{Z}$ for $\mathcal{S}$, while interacting with a real honest party $P_{2}$. The central point here is that since the CA is also corrupted, the adversarial $P_{2}$ can determine the messages that the CA sends to $P_{1}$ in scenario 3 , and make them be exactly the CA functionality messages that $\mathcal{S}$ sends to $\mathcal{Z}$ in scenario 2 (recall that in scenario 2 , $\mathcal{S}$ plays the role of the CA functionality).

The only additional point to prove is that in scenario 2 , the messages generated by the ideal adversary $\mathcal{S}$ when it plays the role of the CA functionality are indistinguishable from the messages that the real honest CA functionality generates (even when viewed together with the entire transcript). However, this is derived from the basic UC definition that guarantees that the environment outputs 1 with at most negligible difference between the real and ideal models. More specifically, if it were true that these messages of $\mathcal{S}$ are distinguishable, then there exists an appropriate polynomial-time distinguisher $D$ that distinguishes them. Now, all that needs to be done is to modify $\mathcal{Z}$ so that at the end of the execution it runs $D$ on the transcript and outputs whatever $D$ outputs. This implies that $\mathcal{Z}$ also distinguishes, in contradiction to the assumed security of the protocol. Given this, $\mathcal{Z}$ can be modified back to the way it was and the proof continues as before.

\section{Universal Composability with Fixed Inputs}

An interesting question that arises in the setting of concurrent composition is how inputs are chosen. As we have mentioned in the introduction, when honest parties choose their inputs adaptively (as a function of previous outputs), concurrent self composition is equivalent to concurrent general composition [27]. Therefore all of the impossibility results that hold for concurrent general composition also hold for concurrent self composition with adaptively chosen inputs. In contrast, when the honest parties' inputs are all fixed ahead of time, there exist functionalities that can be securely computed under concurrent self composition but not under concurrent general composition $[23,3,26]$.

In this section we ask whether or not an analogous situation holds for concurrent general composition. That is, is it possible to achieve concurrent general composition with fixed inputs

\footnotetext{
${ }^{6}$ We note that [15] differentiates between global honest-looking behavior and local honest-looking behavior. In the setting of two-party computation where one party is corrupted, this makes no difference.
} 
in cases that it is impossible where the inputs may be chosen adaptively? To be more exact, we investigate for which functions $f$ it is possible to construct a secure protocol $\pi_{f}$ for the functionality $\mathcal{F}_{f}$ (as defined above) such that $\pi$ remains secure when run concurrently with a single other protocol $\pi^{\prime}$, and when the inputs to $\pi_{f}$ and $\pi^{\prime}$ are fixed before either execution begins.

Defining concurrent general composition with fixed inputs. We define the real and hybrid models exactly as in [26] with the exception that we denote the vector of inputs for $\pi_{f}$ by $\bar{x}_{\pi_{f}}$ and for $\pi^{\prime}$ by $\bar{x}_{\pi^{\prime}}$, and we fix these inputs at the onset. ${ }^{7}$ We denote by $\operatorname{REAL}_{\pi_{f}, \pi^{\prime}, \mathcal{A}}\left(n, \bar{x}_{\pi_{f}}, \bar{x}_{\pi^{\prime}}, z\right)$ a real concurrent execution of protocols $\pi_{f}$ and $\pi^{\prime}$ with adversary $\mathcal{A}$, where $n$ is the security parameter, $\bar{x}_{\pi_{f}}$ is the vector of inputs for the parties in $\pi_{f}, \bar{x}_{\pi^{\prime}}$ is the vector of inputs for the parties in $\pi^{\prime}$, and $z$ is the auxiliary input for the adversary. Likewise, we denote by $\operatorname{HYBRID}_{\pi^{\prime}, \mathcal{S}}^{\mathcal{F}_{f}}\left(n, \bar{x}_{\pi_{f}}, \bar{x}_{\pi^{\prime}}, z\right)$ an execution of the protocol $\pi^{\prime}$ together with an ideal call to $\mathcal{F}_{f}$ that takes the place of the real execution of $\pi_{f}$; the inputs are the same as above. Intuitively, $\pi_{f}$ is secure in this setting if the output distributions of the REAL and HYBRID executions are indistinguishable. We call this minimal concurrent general composition because only two protocol executions take place. We have the following formal definition:

Definition 4.1 Let $\pi_{f}$ be a polynomial-time protocol and let $\mathcal{F}_{f}$ be an ideal functionality. Then, $\pi_{f}$ securely realizes $\mathcal{F}_{f}$ under minimal concurrent general composition with fixed inputs if for every polynomial-time protocol $\pi^{\prime}$ and every probabilistic non-uniform polynomial-time real-model adversary $\mathcal{A}$ for the concurrent executions of $\pi_{f}$ and $\pi^{\prime}$, there exists a probabilistic non-uniform polynomial-time hybrid-model adversary $\mathcal{S}$ such that for all $\bar{x}_{\pi_{f}}, \bar{x}_{\pi^{\prime}} \in\left(\{0,1\}^{*}\right)^{m}$ and $z \in\{0,1\}^{*}$ :

$$
\left\{\operatorname{HYBRID}_{\pi^{\prime}, \mathcal{S}}^{\mathcal{\mathcal { F } _ { f }}}\left(n, \bar{x}_{\pi_{f}}, \bar{x}_{\pi^{\prime}}, z\right)\right\}_{n \in \mathbb{N}} \stackrel{\mathrm{c}}{=}\left\{\operatorname{REAL}_{\pi_{f}, \pi^{\prime}, \mathcal{A}}\left(n, \bar{x}_{\pi_{f}}, \bar{x}_{\pi^{\prime}}, z\right)\right\}_{n \in \mathbb{N}}
$$

where $\stackrel{c}{=}$ denotes computational indistinguishability.

In order to prove our results, we introduce a restricted UC model where the environment first writes the inputs to all parties' input tapes and only once it has finished do the parties begin executing the protocol. Everything else remains the same and so it is possible to define this modification as a restriction on the environments considered if one wishes. A protocol $\pi$ that UC realizes a functionality $\mathcal{F}$ in this model is said to securely realize $\mathcal{F}$ in the UC model with fixed inputs. We first prove the following lemma:

Lemma 4.2 Let $\pi_{f}$ be a polynomial-time protocol and $\mathcal{F}_{f}$ a functionality. If $\pi_{f}$ securely realizes $\mathcal{F}_{f}$ under minimal concurrent general composition with fixed inputs, then $\pi_{f}$ securely realizes $\mathcal{F}_{f}$ in the UC model with fixed inputs.

Proof Sketch: This is proven in almost exactly the same way as the main theorem in [26]. Recall that $\pi_{f}$ is the secure protocol and $\pi^{\prime}$ an arbitrary other protocol. Then, in [26] it is shown that the second protocol $\pi^{\prime}$ can essentially be used to emulate the behavior of the environment $\mathcal{Z}$ in the UC model. This is achieved by $\pi^{\prime}$ defining designated parties $P_{\mathcal{Z}}$ and $P_{\mathcal{A}}$, where $P_{\mathcal{A}}$ is corrupted and $P_{\mathcal{Z}}$ is not. The party $P_{\mathcal{Z}}$ runs the internal code of an environment $\mathcal{Z}$ from the UC setting and $P_{\mathcal{A}}$ does the same for a UC adversary $\mathcal{A}$. Party $P_{\mathcal{Z}}$ sends inputs to the parties (instead of writing them directly on their input tapes as $\mathcal{Z}$ would), receives back their outputs (instead of reading them

\footnotetext{
${ }^{7}$ In $[26]$ there is a distinction between arbitrary and fixed sets of parties. Here we focus on arbitrary sets of parties only, although the analogous results there can be carried through here.
} 
directly from their tapes like $\mathcal{Z}$ would), and interacts with $P_{\mathcal{A}}$ in the same way that $\mathcal{Z}$ interacts with $\mathcal{A}$. Party $P_{\mathcal{A}}$ behaves similarly for the UC-adversary $\mathcal{A}$. The other honest parties in $\pi^{\prime}$ are simply instructed to receive values from $P_{\mathcal{Z}}$ and use them as inputs for the ideal functionality $\mathcal{F}_{f}$ (in the ideal model) or in the protocol $\pi_{f}$ (in the real model). They then send their outputs back to $P_{\mathcal{Z}}$ when they finish. Finally, $P_{\mathcal{Z}}$ outputs the same bit that $\mathcal{Z}$ would output given its view. The crucial point is that it is possible to set up the protocol $\pi^{\prime}$ so that when it is run together with $\mathcal{F}_{f}$ the output of $P_{\mathcal{Z}}$ is distributed identically to the output of $\mathcal{Z}$ in an ideal execution of $\mathcal{F}_{f}$ in the UC model. Furthermore, if $\pi^{\prime}$ is run together with $\pi_{f}$, then the output of $P_{\mathcal{Z}}$ is distributed identically to the output of $\mathcal{Z}$ in a real execution of $\pi_{f}$ in the UC model. The conclusion is therefore that if $\pi_{f}$ is not UC secure, then it is also not secure under concurrent general composition. (This is because when run with $\pi^{\prime}$ the outputs in the REAL and HYBRID distributions will be distinguishable. In particular, $P_{\mathcal{Z}}$ outputs 1 with probability that is non-negligibly different in both.)

The proof here is almost the same with the exception being how the parties' inputs to $\pi_{f}$ are chosen. In [26] these are sent by the party $P_{\mathcal{Z}}$ playing $\mathcal{Z}$ in $\pi^{\prime}$ (and $P_{\mathcal{Z}}$ chooses them by internally running $\mathcal{Z}$ on its auxiliary input $z$ ). However here the inputs must be a priori fixed. In order to do this, first observe that since we are in the UC model with fixed inputs, the environment writes the inputs to the honest parties before any interaction takes place. Thus, by using an averaging argument it is easy to see that if the environment distinguishes the REAL and IDEAL executions with non-negligible probability in the UC model with fixed inputs, then there exists a vector of inputs $\bar{x}_{\pi_{f}}$ for which the environment distinguishes the REAL and IDEAL with non-negligible probability. (Note that the environment may choose the inputs as a function of its auxiliary input $z$ and its random coins. Thus, the averaging argument is needed to fix a specific input vector. Note also that this works only because $\mathcal{Z}$ determines the parties' inputs based on its auxiliary input and own random tape only; in particular, the inputs are determined independently of the honest parties' random tapes.) Next, party $P_{\mathcal{Z}}$ 's input in $\pi^{\prime}$ is set to the value $z$ and random-tape for $\mathcal{Z}$ that results in the environment writing $\bar{x}_{\pi_{f}}$. Furthermore, all the parties' inputs in $\pi_{f}$ are set to $\bar{x}_{\pi_{f}}$. When the inputs are set in this way, the execution in the setting of concurrent general composition perfectly emulates the setting of the UC model with fixed inputs (when $\mathcal{Z}$ has the appropriate $z$ and random tape). More specifically, the output of $P_{\mathcal{Z}}$ in a REAL execution of $\pi_{f}$ with $\pi^{\prime}$ will be distributed exactly like the output of the environment $\mathcal{Z}$ in a real execution in the UC model with fixed inputs (when $\mathcal{Z}$ has the appropriate $z$ and random tape). Likewise, the output in a HYBRID execution of $\pi^{\prime}$ with $\mathcal{F}_{f}$ will be exactly like an ideal execution in the UC model with fixed inputs. Thus, $P_{\mathcal{Z}}$ will distinguish the REAL and HYBRID models with the same probability that $\mathcal{Z}$ distinguishes the REAL and IDEAL executions in the UC model with fixed inputs. We conclude that if $\pi_{f}$ is not secure in the UC model with fixed inputs then it is not secure under minimal concurrent general composition with fixed inputs. This implies the lemma.

We note that the above lemma holds as long as the order of quantifiers between $\mathcal{S}$ and $\mathcal{Z}$ can be switched (as is the case with the definition of universal composability in the latest version of [8]). Otherwise, minimal concurrent general composition only implies a weaker form of universal composability. In any case, the known impossibility results all hold for this weaker form and so for our purposes here there is no difference. See [26] for more discussion on this (note that the issues that arise here and in $[26]$ in this respect are exactly the same).

We are now ready to prove our impossibility results, and we begin once again with an analogue to Lemma 3.1.

Lemma 4.3 Let $f$ be a polynomial-time two-party function, and let $\mathcal{F}_{f}$ be the two-party ideal functionality that receives $x_{1}$ from $P_{1}$ and $x_{2}$ from $P_{2}$, and hands them back their respective outputs 
$f_{1}\left(x_{1}, x_{2}\right)$ and $f_{2}\left(x_{1}, x_{2}\right)$. If $\mathcal{F}_{f}$ can be securely realized in the UC model with fixed inputs by a non-trivial protocol $\pi_{f}$ (in the plain model or in the $\mathcal{F}_{\mathrm{BBCA}}, \mathcal{F}_{\mathrm{UKCA}}$ or $\mathcal{F}_{\mathrm{BPK}}$-hybrid models), then there exists a machine $P_{2}^{a}$ such that for every machine $P_{2}^{b}$ of the form described in Definition 2.2, the split adversarial strategy for $P_{2}=\left(P_{2}^{a}, P_{2}^{b}\right)$ is successful, except with negligible probability.

Proof: Note that in the proof of Lemma 3.1, the environment $\mathcal{Z}$ chooses the inputs for $P_{1}$ and $P_{2}$ at the onset. Thus, in the UC model with fixed inputs the entire proof goes through without change. (One small technicality is that the actual $P_{1}$ should not receive $x_{1}$ since this is the input used by $\mathcal{Z}$ who plays $P_{1}$. Thus, $x_{2}$ can be written to $P_{2}$ 's input tape at the onset, and $\mathcal{Z}$ can write the all-zero string to $P_{1}$ 's input tape. In this way, $x_{1}$ is kept secret by $\mathcal{Z}$ who uses it in its computation.)

Next, we use the following observation:

All of the impossibility results of [12] that use successful split adversarial strategies hold even for the UC model with fixed inputs.

Note that unlike the impossibility results from Section 3, we need the above observation because the impossibility results of [12] were proven for the standard UC model, and not for the variant with fixed inputs. Combining Lemmas 4.2 and 4.3 with this observation, we conclude that there exist large classes of functionalities for which it is impossible to achieve minimal concurrent general composition with fixed inputs.

We stress that this impossibility result is extremely strong. It is not possible to achieve concurrent general composition even when only two protocol executions take place and even when the inputs to these executions are a priori fixed.

\section{Acknowledgements}

We would like to thank the anonymous referees for their helpful comments.

\section{References}

[1] B. Barak, R. Canetti, Y. Lindell, R. Pass and T. Rabin. Secure Computation Without Authentication. In CRYPTO 2005, Springer-Verlag (LNCS 3621), pages 361-377, 2005.

[2] B. Barak, R. Canetti, J. Nielsen and R. Pass. Universally Composable Protocols with Relaxed Set-up Assumptions. In 45th FOCS, pages 186-195, 2004.

[3] B. Barak, M. Prabhakaran and A. Sahai. Concurrent Non-Malleable Zero-Knowledge. In 47th FOCS, pages 345-354, 2006.

[4] B. Barak and A. Sahai How To Play Almost Any Mental Game Over The Net - Concurrent Composition via Super-Polynomial Simulation. In 46th FOCS, pages 543-552, 2005.

[5] D. Beaver. Foundations of Secure Interactive Computing. In CRYPTO'91, Springer-Verlag (LNCS 576), pages 377-391, 1991.

[6] M. Ben-Or, S. Goldwasser and A. Wigderson. Completeness Theorems for NonCryptographic Fault-Tolerant Distributed Computation. In 20th STOC, pages 1-10, 1988. 
[7] R. Canetti. Security and Composition of Multiparty Cryptographic Protocols. Journal of Cryptology, 13(1):143-202, 2000.

[8] R. Canetti. Universally Composable Security: A New Paradigm for Cryptographic Protocols. In 42nd FOCS, pages 136-145, 2001.

[9] R. Canetti. Universally Composable Signature, Certification, and Authentication. In the 17th Computer Security Foundations Workshop, pages 219-235, 2004.

[10] R. Canetti and M. Fischlin. Universally Composable Commitments. In CRYPTO 2001, Springer-Verlag (LNCS 2139), pages 19-40, 2001.

[11] R. Canetti, O. Goldreich, S. Goldwasser and S. Micali. Resettable Zero-Knowledge. In 32nd STOC, pages 235-244, 2000.

[12] R. Canetti, E. Kushilevitz and Y. Lindell. On the Limitations of Universal Composable Two-Party Computation Without Set-Up Assumptions. Journal of Cryptology, 19(2):135$167,2006$.

[13] D. Chaum, C. Crépeau and I. Damgård. Multi-party Unconditionally Secure Protocols. In 20th STOC, pages 11-19, 1988.

[14] R. Canetti, Y. Lindell, R. Ostrovsky and A. Sahai. Universally Composable Two-Party and Multi-Party Computation. In 34th STOC, pages 494-503, 2002.

[15] R. Canetti and R. Ostrovsky. Secure Computation with Honest-Looking Parties: What If Nobody Is Truly Honest? In the 31st STOC, pages 255-264, 1999.

[16] I. Damgård, J.B. Nielsen and C. Orlandi. On the Necessary and Sufficient Assumptions for UC Computation. In the 7th TCC, Springer-Verlag (LNCS 5978), pages 109-127, 2010.

[17] A. Datta, A. Derek, J.C. Mitchell, A. Ramanathan and A. Scedrov. Games and the Impossibility of Realizable Ideal Functionality. In the 3rd TCC, Springer-Verlag (LNCS 3876), pages $360-379,2006$.

[18] Y. Deng, G.D. Crescenzo and D. Lin. Concurrently Non-Malleable Zero Knowledge in the Authenticated Public-Key Model. Cryptology ePrint Archive, Report \#2006/314, 2006.

[19] O. Goldreich. Foundations of Cryptography: Volume 2-Basic Applications. Cambridge University Press, 2004.

[20] O. Goldreich, S. Micali and A. Wigderson. How to Play any Mental Game - A Completeness Theorem for Protocols with Honest Majority. In 19th STOC, pages 218-229, 1987.

[21] S. Goldwasser and L. Levin. Fair Computation of General Functions in Presence of Immoral Majority. In CRYPTO’90, Springer-Verlag (LNCS 537), pages 77-93, 1990.

[22] Y. Kalai, Y. Lindell and M. Prabhakaran. Concurrent General Composition of Secure Protocols in the Timing Model. In the 37th STOC, pages 644-653, 2005.

[23] E. Kushilevitz, Y. Lindell and T. Rabin. Information-Theoretically Secure Protocols and Security Under Composition. In 38th STOC, pages 109-118, 2006. 
[24] H. Lin, R. Pass and M. Venkitasubramaniam. A Unified Framework for Concurrent Security: Universal Composability from Stand-Alone Non-Malleability. In the 41st STOC, pages 179$188,2009$.

[25] Y. Lindell. Composition of Secure Multi-Party Protocols - A Comprehensive Study. Lecture Notes in Computer Science Vol. 2815, Springer-Verlag, 2003.

[26] Y. Lindell. General Composition and Universal Composability in Secure Multi-Party Computation. In 44th FOCS, pages 394-403, 2003.

[27] Y. Lindell. Lower Bounds for Concurrent Self Composition. In the 1st Theory of Cryptography Conference (TCC), Springer-Verlag (LNCS 2951), pages 203-222, 2004.

[28] S. Micali and P. Rogaway. Secure Computation. Unpublished manuscript, 1992. Preliminary version in CRYPTO'91, Springer-Verlag (LNCS 576), pages 392-404, 1991.

[29] R. Ostrovsky, G. Persiano and I. Visconti. Concurrent Non-Malleable Witness Indistinguishability and its Applications. Cryptology ePrint Archive, Report \#2006/256, 2006.

[30] R. Pass. Simulation in Quasi-Polynomial Time, and Its Application to Protocol Composition. In Eurocrypt 2003, Springer-Verlag (LNCS 2656), pages 160-176, 2003.

[31] M. Prabhakaran and A. Sahai. New Notions of Security: Universal Composability Without Trusted Setup. In 36th STOC, pages 242-251, 2004.

[32] A. Yao. How to Generate and Exchange Secrets. In 27th FOCS, pages 162-167, 1986. 